\title{
A Multicentre, Double-Blind, Placebo-Controlled, Parallel-Group Study to Evaluate the Efficacy, Safety, and Tolerability of the S1P Receptor Agonist KRP203 in Patients with Moderately Active Refractory Ulcerative Colitis
}

\author{
Heinfried H. Radekea,b Jürgen Stein ${ }^{a}$ Gert Van Assched Gerhard Rogler ${ }^{f}$ \\ Peter L. Lakatos ${ }^{9}$ Florian Muellershausen ${ }^{\mathrm{e}}$ Pierre Moulin $^{\mathrm{e}}$ Philip Jarvis $^{\mathrm{e}}$ \\ Laurence Colin $^{\mathrm{e}}$ Peter Gergely ${ }^{\mathrm{e}}$ Wolfgang Kruis ${ }^{\mathrm{c}}$ \\ anterdisciplinary Crohn Colitis Centre Rhein-Main, Frankfurt am Main, Germany; ${ }^{b}$ Hospital of the Goethe University

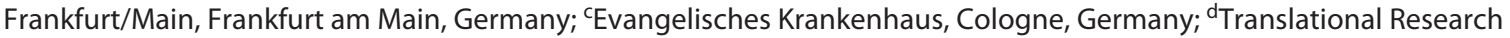 \\ in Gastrointestinal Disorders, School of Medicine, University of Leuven, Leuven, Belgium; 'Novartis Basel, Basel, \\ Switzerland; ${ }^{f}$ Clinic of Gastroenterology and Hepatology, University Hospital Zurich, University of Zurich, Zurich, \\ Switzerland; 9IBD Centre, Department of Medicine, McGill University, Montreal, QC, Canada
}

\section{Keywords}

Clinical trials · Ulcerative colitis · Sphingosine-1-phosphate

\begin{abstract}
Background and Aims: KRP203 is a potent oral agonist of the sphingosine-1-phosphate receptor subtype 1 that induces the sequestration of peripheral lymphocytes, thereby potentially reducing the number of activated lymphocytes circulating to the gastrointestinal tract. Methods: We conducted a multicentre, double-blind, placebo-controlled, parallel-group, proof-of-concept study to evaluate the efficacy, safety, and tolerability of KRP203 in patients with moderately active 5-aminosalicylate-refractory ulcerative colitis (UC). Patients were randomly assigned to receive $1.2 \mathrm{mg} \mathrm{KRP203}$ or placebo daily for 8 weeks. Primary efficacy variable was clinical remission, defined as partial Mayo Score 0-1 and modified Baron Score 0-1 with rectal bleeding subscore 0. Results: KRP203 was safe and well tolerated overall. The most common adverse events (AEs) were gastrointestinal
\end{abstract}

karger@karger.com www.karger.com/iid

Karger $\frac{1}{\%}$

GOPEN ACCESS
(C) 2020 The Author(s)

Published by S. Karger AG, Basel

This article is licensed under the Creative Commons AttributionNonCommercial-NoDerivatives 4.0 International License (CC BYNC-ND) (http://www.karger.com/Services/OpenAccessLicense). Usage and distribution for commercial purposes as well as any distribution of modified material requires written permission. disorders and headache. Importantly, no KRP203-related cardiac AEs were reported. Total peripheral lymphocytes and selectively affected lymphocyte subtypes decreased, causing marked decreases in naive and central memory CD4+ and CD8+ T cells, and also in B cells. Clinical remission occurred in 2/14 (14\%) patients under KRP203, compared with 0/8 (0\%) under placebo. Conclusions: Overall, KRP203 was safe and well tolerated by patients with UC. Importantly, no cardiac AEs were reported. Although KRP203 did not meet the minimum clinically relevant threshold for efficacy, the results may suggest that KRP203 treatment is superior to placebo. However, in this small study population, the difference was insignificant. Based on these data, studies with an improved design and a larger population should be considered.

(c) 2020 The Author(s)

Published by S. Karger AG, Basel

H.H.R. and W.K. contributed equally.

Clinical Trial Protocol No. CKRP203A2201; Eudra-CT No. 2010-019970-

33; Clinicaltrials.Gov Identifier NCT01375179. 


\section{Introduction}

Ulcerative colitis (UC) is a chronic immune-mediated disorder that involves inflammation and ulceration in the gastrointestinal tract, resulting in diarrhoea, abdominal pain, and rectal bleeding. Conventional oral immunomodulators (e.g., thiopurines and methotrexate) are commonly used to treat patients with UC but are limited in efficacy and often have a delayed-onset action, while biologics (e.g., anti-tumour necrosis factor [anti-TNF] antibodies) can be effective but are associated with serious adverse reactions including opportunistic infections and lymphoma. Thus, there is a substantial unmet medical need for oral therapies of UC, which are highly effective, safe, and well tolerated.

The recirculation and infiltration (homing) of autoreactive lymphocytes into the gut mucosa (lamina propria) is involved in the pathogenesis of inflammatory bowel disease. Lymphocyte trafficking has therefore been identified as a rational target to ameliorate these immunopathologic processes. Studies of monoclonal antibodies directed toward adhesion molecules have previously shown that the interruption of lymphocyte trafficking is an effective therapeutic approach for patients with UC. The use of orally administered small molecules as alternatives to injectable monoclonal antibodies for the treatment of UC would avoid sensitisation and the formation of antidrug antibodies and could thus potentially eliminate one of the most important reasons for treatment failure with monoclonal antibodies.

The sphingosine-1-phosphate (S1P) subtype 1 (S1P1) receptor is a member of a family of 5 receptors (S1P1S1P5) responsible for regulating basic cellular and multiple immunologic and cardiovascular effects $[1,2]$. Cell surface-associated S1P1 receptor plays a crucial role in the trafficking of lymphocytes from lymphoid organs [3]. $\mathrm{S} 1 \mathrm{P} 1$ receptor agonists induce internalisation and degradation of the S1P1 receptor, rendering $\mathrm{B}$ and $\mathrm{T}$ lymphocytes incapable of emigrating from secondary lymphoid organs, which leads to a reversible reduction in circulating lymphocytes in the blood [2].

Fingolimod, a non-selective S1P receptor modulator, has been approved for the treatment of relapsing multiple sclerosis but has a number of safety issues including cardiovascular events (e.g., bradycardia, conduction abnormalities, and hypertension), macular oedema, and elevated liver transaminases [4]. Most recently, ozanimod, a selective and potent partial agonist of S1P1R and S1P5R, has been demonstrated to have a promising safety and efficacy profile [5].

S1P Receptor Agonist KRP203 in

Ulcerative Colitis
KRP203 (2-amino-2-1,3-propanediol hydrochloride) is a synthetic, second generation oral S1P receptor modulator discovered and synthesised by Kyorin Pharmaceutical Co., Japan. KRP203 is a prodrug, which is phosphorylated in vivo by the sphingosine kinase 2 . The $(+)$ enantiomer of the phosphate metabolite, (+)KRP203 phosphate, is the active entity and targets the S1P1 receptor. KRP203 displays only partial agonism at the human S1P3 receptor, hereby supposedly reducing the risk of bradycardia [6].

In preclinical models of transplantation $[7,8]$ and IL10-knockout mouse colitis [9], orally administered KRP203 led to the sequestration of lymphocytes into secondary lymphoid organs and reduced lymphocyte counts in the blood, but also, more importantly, in the intestine. Due to this major inhibitory effect on lymphocyte recruitment, oral KRP203 may have beneficial effects in patients with UC. Subsequently, a reversible and dose-dependent reduction of blood lymphocyte (absolute lymphocyte count $[\mathrm{ALC}]$ ) levels was demonstrated in phase I clinical trials (Novartis, unpublished). This is believed to be caused by functional antagonism at the S1P1 receptor on lymphocytes such as $\mathrm{T}$ and $\mathrm{B}$ cells, due to persistent ligand-induced internalisation of the S1P1 receptor [10].

The novel, oral immunomodulator KRP203 may represent an alternative to the current second- or third-line treatments in UC. The purpose of this study was to characterise the safety, pharmacokinetics (PK), pharmacodynamics (PD), and efficacy of KRP203 for induction of remission in patients with moderately active UC.

\section{Patients and Methods}

This phase IIa, randomised, double-blind, placebo-controlled, parallel-group study was designed to evaluate the efficacy, safety, and tolerability of KRP203 in patients with moderately active UC. Study participants were recruited from November 2010 to August 2012 from 9 centres in Belgium, Switzerland, Hungary, Sweden, and Germany. The protocol was approved by the Ethics Committee of the Ärztekammer Nordrhein and the institutional review board responsible for each centre. The trial was conducted in accordance with the Declaration of Helsinki and the ethical principles of Good Clinical Practice according to the International Conference on Harmonization (ICH) Harmonized Tripartite Guideline. The trial is registered at Clinicaltrials.gov (identifier 2010-019970-33). All patients were personally informed about all aspects of the trial including possible risks of treatment and provided written informed consent prior to screening.

Patients were enrolled and randomly assigned to KRP203 (with dose titration as described below) or identical placebo at a 2:1 ratio, stratified according to steroid treatment at baseline (yes/no). Each patient underwent a screening period of up to 28 days (day-35 to day-8, Visit 1), during which a full physical examination was per-

Inflamm Intest Dis 2020;5:180-190 181 
formed, vital signs (Fig. 1) were recorded, and relevant medical history and concomitant medication were documented. At the baseline visit (day-7 to day-1 \pm 1 , Visit 2), sigmoidoscopy with biopsy collection and all other required laboratory and safety evaluations including lung function and optical examinations (visual acuity, ophthalmoscopy, and optical coherence tomography) were performed. In addition, at Visits 1 and 2, pregnancy testing was performed in females of childbearing age. Randomisation and treatment initiation with PK sampling were performed at Visit 3 (day 1). Study medication was administered thereafter orally once daily, preferably with a light breakfast, before 12:00 noon at the latest. Patients returned to the study centre before taking their morning dose on day $7 \pm 1$ (Visit 4), day $15 \pm 1$ (Visit 5), day $28 \pm$ 3 (Visit 6), and day $56 \pm 3$ (Visit 7) for study assessments. The primary end point was clinical remission at the week 8 visit (Visit 7).

Approximately 72 patients were intended to be randomised into the study, and after 30 patients had completed the 8-week treatment period with KRP203 or placebo, an interim analysis (IA) was to be performed to determine the preliminary efficacy. The investigator was blinded to certain assessments (mentioned below) to avoid potential bias based upon insight regarding active versus placebo treatment. A separate co-investigator or designee reviewed ALC, electrocardiograms, and mobile cardiac output telemetry, performed from baseline (day-1) to day $12 \pm 1$. Patients were allowed to continue to take 5-aminosalicylates (5-ASAs) and oral corticosteroids (maximum $40 \mathrm{mg}$ /day prednisone or equivalent) at the dosage administered at baseline, as per standard medical practice. After 2 weeks of treatment (Visit 5), if deemed necessary by the primary investigator, corticosteroid dose tapering was permitted.

Concomitant treatment with intravenous or rectal steroids, 5-ASA rectal suppositories, class III antiarrhythmic drugs or insulin for treatment of diabetes, or any biologic (e.g., anti-TNF) or immunosuppressive (e.g., azathioprine, 6-mercaptopurine, and methotrexate) drug during the study was not permitted. Administration of acetaminophen was allowed (online suppl. 1; for all online suppl. material, see www.karger.com/doi/10.1159/000509393).

\section{Patients}

The study population comprised patients with clinically active UC who had shown inadequate response to conventional therapy with oral 5-ASA prior to screening. Male and female patients 1865 years of age with active disease, as indicated by a partial Mayo Score of 5-9, with a score of at least 2 for either stool frequency or rectal bleeding and a modified Baron Score of at least 2 upon endoscopic examination, and with disease extending at least $25 \mathrm{~cm}$ from the anal verge, were enrolled. Baron scores were evaluated locally by the investigator on the basis of the screening endoscopy. Patients were allowed to continue concomitant therapy with 5 -ASAs and/or oral corticosteroids ( $\max 40 \mathrm{mg}$ /day prednisone or equivalent) during the study. For study inclusion/exclusion criteria, see online Suppl. 1.

\section{Study Drug}

Dosage of KRP203, ranging from 0.3 to $1.2 \mathrm{mg} /$ day, was derived from the results of the CKRP-203A2101 and CKRP-203A2102 studies. The starting dose was $0.3 \mathrm{mg} / \mathrm{day}$, a dosage that had been found to have no, or only a minimal, PD effect inducing bradycardia and decrease of ALC. The therapeutic dose was projected to be $1.2 \mathrm{mg} /$ day, associated with an ALC reduction of $\sim 75 \%$ after 2

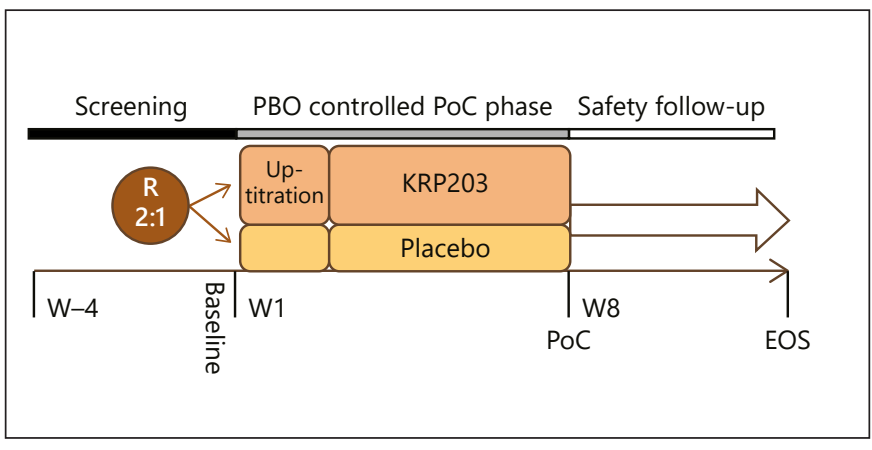

Fig. 1. Trial schematics. $\mathrm{PBO}$, placebo; PoC, point of care, $\mathrm{R}$, randomisation; $\mathrm{W}$, week.

weeks of treatment. The present study used a 12-day dose titration scheme employing doses of $0.3 \mathrm{mg} /$ day (days $1-4), 0.6 \mathrm{mg} /$ day (days 5-8), and $0.9 \mathrm{mg} /$ day (days 9-12). Study drug (KRP203 0.1, 0.4 , and $1 \mathrm{mg}$ ) and matching placebo capsules were prepared by Novartis and supplied in bottles by Novartis Drug Supply Management to the investigator as semi-bulk medication.

Serial blood samples were collected from subjects for measurement of ALC and assessment of changes from baseline. Blood samples were collected at baseline $(-24,-22,-20,-18,-16,-12$, and $-8 \mathrm{~h}$ and immediately predose), on day $1(2,4,6,8,12$, and $16 \mathrm{~h}$ postdose), and on day 2 (at 24 and 36 h postdose).

\section{Efficacy Assessment}

The following efficacy assessments were performed: partial Mayo Score, modified Baron Score as evaluated locally by the investigator on the basis of sigmoidoscopy, faecal calprotectin, erythrocyte sedimentation rate, C-reactive protein (CRP), and ALC. Changes in ALC from baseline were calculated and displayed graphically according to time point and treatment group. The course of the partial Mayo and modified Baron scores over time was also recorded and displayed according to treatment group.

The primary efficacy variable, clinical remission, was defined as a partial Mayo Score of 0 or 1 and a modified Baron Score of 0 or 1 with no macroscopic bleeding (partial Mayo subscore of 0 for rectal bleeding). The Baron Score, first described in 1964 [11], is an endoscopic index of mucosal healing that is based on visual assessment of the bowel mucosa during endoscopy, on a scale ranging from 0 to 4 , where 0 denotes normal mucosa, 1 granular mucosa with an abnormal vascular pattern, 2 friable mucosa, 3 microulceration with spontaneous bleeding, and 4 gross ulceration.

The change from baseline in the partial Mayo (clinical activity) score at week 8 was considered a key secondary end point. Partial response was defined as a reduction of at least 3 points in the partial Mayo score from baseline to week 8 . Reduction in corticosteroid dose after 2 weeks was considered another secondary end point.

\section{Safety Assessments}

Safety assessments included all adverse events (AEs) and severe adverse events (SAEs), which were recorded along with their severity and relationship to study drug, as assessed by the investigator. Pregnancies and their outcomes were also documented. Laboratory assessments were evaluated at a central laboratory routinely 
including haematology, blood chemistry, stool tests, and urine panel, while vital signs, clinical symptoms, and body weight were regularly assessed and documented at the study visits. Other safety assessments were serology testing, mobile cardiac output telemetry, electrocardiograms, ophthalmic examination, and pregnancy status.

Detailed information concerning the AEs was tabulated according to the treatment group and patient. The number of AEs and the percentage of patients in whom they occurred were classified according to the affected system organ class (SOC) and preferred terminology, and stratified according to the treatment group. A patient with multiple AEs within a specific SOC was counted only once toward the total of this SOC. Individual patients were withdrawn in case of clinically significant abnormal laboratory values, for example, lymphocyte count below 200 cells $/ \mu \mathrm{L}$ or 200 cells $/ \mathrm{mm}^{3}$ in 2 consecutive measurements, Mobitz type II atrioventricular (AV) block, or bradycardia $<40 \mathrm{bpm}$.

\section{Statistical Analysis}

Demographic characteristics and disease characteristics at baseline were summarised using descriptive statistics. An IA was to be performed after 30 patients had completed 8 weeks of treatment with KRP203 or placebo to identify whether dual efficacy criteria had been met. The dual efficacy criteria were designed to take into account both observed effect size and evidence for superiority against placebo [12]. Study continuation was to depend on an IA showing 20\% superiority of KRP203 over placebo. Bayesian posterior probabilities were calculated for 2 hypotheses of interest: $\mathrm{H} 0$, no effect and $\mathrm{H} 1$, minimal relevant effect. $\mathrm{H} 0$ corresponded to a remission rate under KRP203 not greater than that under placebo, while $\mathrm{H} 1$ corresponded to a remission rate at least 20 percentage points superior to that detected under placebo.

The study was to be considered a positive sign for efficacy if the posterior probability against $\mathrm{H} 0$ were $95 \%$ or more and the posterior probability in favour of $\mathrm{H} 1$ at least $50 \%$ in the IA or at study completion. The prior probability distribution for placebo drew information worth 20 patients from previous studies in a comparable patient population [13-15]. The historical placebo clinical remission rate was estimated to be $12 \%$, making a prior Beta distribution of $(2.4,17.6)$. For the KRP203 prior distribution, a "flat" non-informative probability distribution was assumed (Beta (1, 1)). The overall information from this unevenly randomised trial together with these prior distributions was balanced between the 2 treatment groups.

The sample size of 60 complete patient data sets was determined based on historical data to allow for a robust comparison between $\mathrm{H} 0$ and $\mathrm{H} 1$. Simulations showed the probability of meeting the above criteria under $\mathrm{H} 0$ at the IA or (if the trial continued) at final analysis to be below 5\%. The probability of meeting these efficacy criteria at the IA or (if the trial continued) at final analysis was determined to be approximately 65,92 , or $100 \%$, assuming a true difference in remission probabilities of 20,30 , or $50 \%$ points, respectively, and a probability of true placebo remission of $12 \%$, in line with historical data.

Tolerability was assessed on the basis of the number and percentage of patients who failed to complete the study, and the number of patients who failed to complete the study due to AEs. All patients who completed at least 28 days of treatment (i.e., visit 6) were included in the primary efficacy analysis. Patients who dis- continued after at least 28 days of treatment due to lack of efficacy were considered non-responders.

Data were collected by a contract research organisation (Pharmaceutical Product Development) and analysed by the sponsor. The sponsor and the steering committee interpreted the data jointly.

\section{Results}

\section{Patient Disposition and Baseline Characteristics}

Approximately 72 patients were intended to be randomised in the study, and a preliminary efficacy analysis was planned after 30 patients had completed the 8 -week treatment period with $1.2 \mathrm{mg}$ KRP203 or placebo. Twenty-seven patients were randomised and stratified according to baseline corticosteroid intake (yes/no). A total of 22 patients who received $>28$ days therapy were evaluable for the efficacy analysis (Fig. 2). A total of 12 patients discontinued the study prematurely ( 7 out of 17 in the KRP203 group, 5 out of 10 in the placebo group) mostly due to inadequate therapeutic response (5 KRP203, 4 placebo). All 27 patients were included in the safety and PD analysis set. The overall mean age of the 27 enrolled patients was 37 years (range: 19-65 years). Fifteen $(55.6 \%)$ males and $12(44.4 \%)$ females were enrolled, with a mean height of $170.5 \mathrm{~cm}$ (range: 155-182 cm), a mean weight of $70.26 \mathrm{~kg}$ (range: $49-102.9 \mathrm{~kg}$ ), and a mean BMI of 24.15 $\mathrm{kg} / \mathrm{m}^{2}$ (range: $17.7-37.6 \mathrm{~kg} / \mathrm{m}^{2}$ ). Other baseline characteristics are summarised in Table 1. At baseline, the mean partial Mayo Score was 7.7 in the KRP203 group and 8.0 in the placebo group. The mean modified Baron Score was 3.1 in the active group and 2.8 in the placebo group, indicating that disease severity at baseline was comparable for the 2 cohorts.

\section{Safety Analysis}

No clinically significant changes were observed in body weight, temperature, blood pressure, or pulse rate during the course of the study. Of the 27 patients enrolled, $24(88.9 \%)$ experienced at least one AE. In patients exposed to both KRP203 and placebo, gastrointestinal (GI) disorders were identified as the most commonly affected SOC, with an overall incidence of $40.7 \%$. This was followed by nervous system disorders (25.9\%), general disorders and administration site conditions (22.2\%), and musculoskeletal and connective tissue disorders (22.2\%). In the KRP203 group, there was a higher incidence of general disorders and administration site conditions ( 29.4 vs. $10.0 \%$ in the placebo group) and a slightly higher rate of infections ( 23.5 vs. $10 \%$ in the placebo group). 


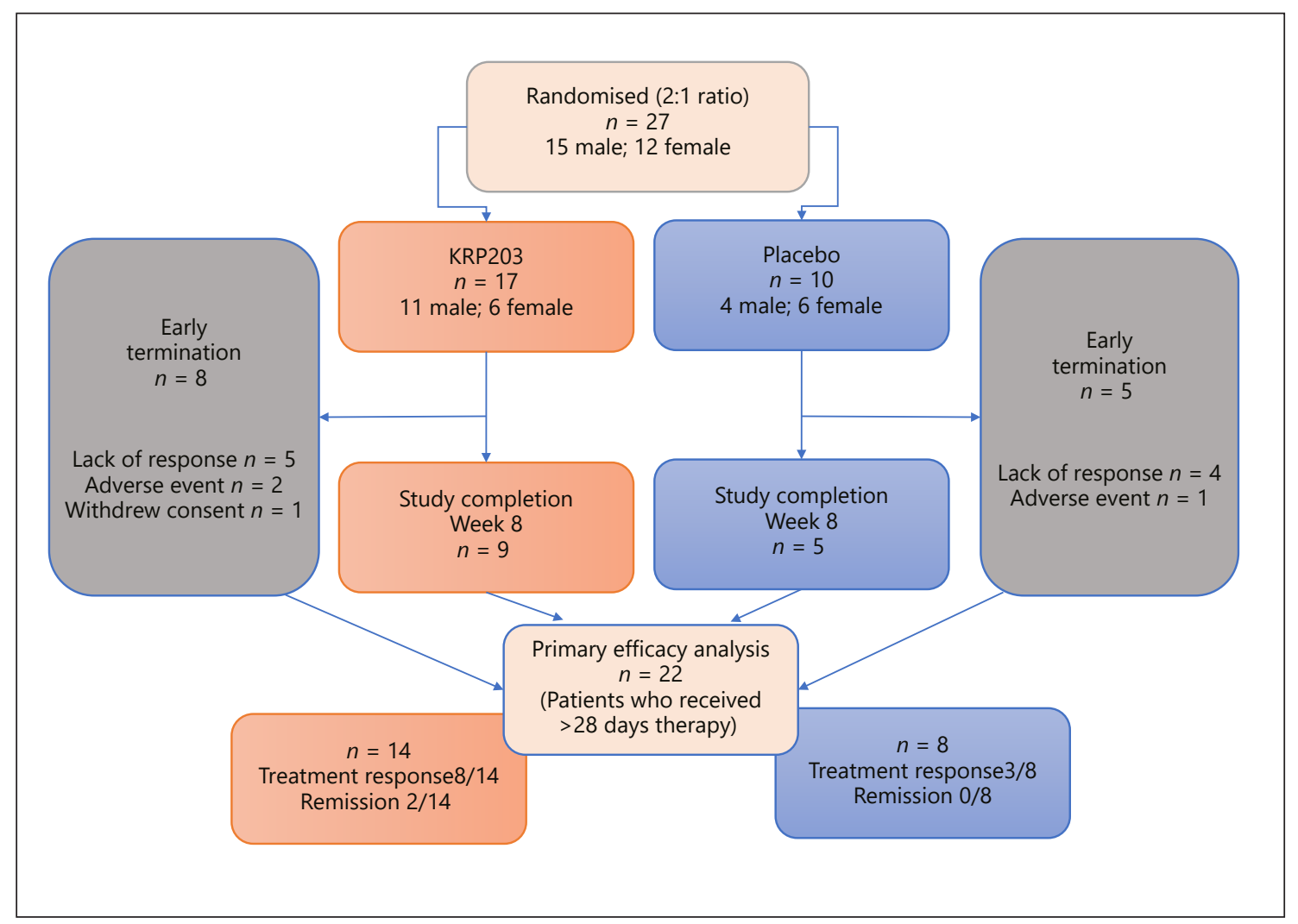

Fig. 2. Flow chart of study participants. For early terminations, the number of patients terminated due to lack of response, adverse events, or withdrawal of consent, respectively, is shown.

However, these differences are based on small numbers of patients ( 5 or less). The summary of AEs is presented in Table 2. While headache (23.5\%) was the most commonly reported individual $\mathrm{AE}$ in patients receiving treatment with KRP203, headache (20\%) and diarrhoea (20\%) were the most commonly reported individual AEs in the placebo group. Overall, the reported AEs were evenly distributed in frequency in both treatment groups. One female patient discontinued study participation due to lymphopenia (abnormal laboratory values) on day 14, suspected to be related to KRP203. The event resolved. Three patients were reported to have experienced eye-related AEs of conjunctival and ocular hyperaemia. These events also resolved.

In total, 7 SAEs were reported during the study. Two of these occurred in 2 patients in the KRP203 treatment group: infectious diarrhoea requiring hospitalisation was reported in a 22-year-old Caucasian female, reported as moderate in intensity and adjudged by the investigator to be possibly related to the study drug. The study drug was interrupted for 10 days and the event resolved without sequelae. In the second case, severe osteoarthritis was reported in a 57-year-old Caucasian male with a history of arthrosis and osteoporosis. The SAE resolved after surgery, without treatment interruption, and the event was adjudged by the investigator to be not related to the study drug.

The remaining 5 SAEs were reported in 2 patients in the placebo arm: in a 20-year-old female patient of colour, severe haemorrhagic diarrhoea or severe diarrhoea was reported at total of 4 times as separate SAEs requiring hospitalisation. The remaining SAE was a diagnosis of vena cava thrombosis in a 28 -year-old Caucasian female, requiring hospitalisation and ongoing at the time of discontinuation.

In total, 3 patients discontinued study participation due to AEs: 2 patients in the KRP203 group with AEs suspected to be related to the study medication and 1 patient in the placebo group. Importantly, no cases of macular oedema were observed during the trial. In addition, the frequency of bradycardia or other cardiac abnormalities was no higher in the KRP203 than in the placebo group, 
Table 1. Demographic and disease characteristics according to treatment group

\begin{tabular}{|c|c|c|c|}
\hline & $\begin{array}{l}\text { KRP203 } 1.2 \mathrm{mg} \\
n=17\end{array}$ & $\begin{array}{l}\text { Placebo } \\
n=10\end{array}$ & $\begin{array}{l}\text { Total } \\
n=27\end{array}$ \\
\hline \multicolumn{4}{|l|}{ Age, years } \\
\hline Mean (SD) & $40.1(16.23)$ & $31.7(8.53)$ & $37.0(14.29)$ \\
\hline Median & 37.0 & 28.5 & 36.0 \\
\hline Range & 19,65 & 20,46 & 19,65 \\
\hline \multicolumn{4}{|l|}{ Sex, $n(\%)$} \\
\hline Male & $11(64.7)$ & $4(40.0)$ & $15(55.6)$ \\
\hline Female & $6(35.3)$ & $6(60.0)$ & $12(44.4)$ \\
\hline \multicolumn{4}{|l|}{$\mathrm{BMI}, \mathrm{kg} / \mathrm{m}^{2}$} \\
\hline Mean (SD) & $23.452(3.20)$ & $25.34(5.76)$ & $24.153(4.3190)$ \\
\hline Median & 23.301 & 25.152 & 23.356 \\
\hline Range & $18.90,31.76$ & $17.75,37.63$ & $17.75,37.63$ \\
\hline \multicolumn{4}{|l|}{ Race, $N(\%)$} \\
\hline Caucasian & $16(94.1)$ & $9(90.0)$ & $25(92.6)$ \\
\hline Black & & $1(10.0)$ & $1(3.7)$ \\
\hline Other & $1(5.9)$ & & $1(3.7)$ \\
\hline \multicolumn{4}{|c|}{ Partial Mayo Score at baseline $\mathrm{a}^{\mathrm{a}}$} \\
\hline Mean (SD) & $7.7(1.21)$ & $8.0(0.94)$ & $7.8(1.11)$ \\
\hline Median & 8.0 & 8.0 & 8.0 \\
\hline Range & {$[6 ; 11]^{\mathrm{b}}$} & {$[6 ; 9]$} & {$[6 ; 11]^{\mathrm{b}}$} \\
\hline \multicolumn{4}{|c|}{ Modified Baron Score at baseline } \\
\hline Mean (SD) & $3.1(0.83)$ & $2.8(0.63)$ & $3.0(0.76)$ \\
\hline Median & 3.0 & 3.0 & 3.0 \\
\hline Range & {$[2 ; 4]$} & {$[2 ; 4]$} & {$[2 ; 4]$} \\
\hline \multicolumn{4}{|c|}{ Baseline intake of steroids: prednisone equivalent dose at baseline, $\mathrm{mg}$} \\
\hline$N(\%)$ & $11(64.7)$ & $6(60.0)$ & $17(63.0)$ \\
\hline Mean (SD) & $16.9(6.89)$ & $13.8(13.67)$ & $15.6(9.82)$ \\
\hline Median & 15.0 & 10.0 & 15.0 \\
\hline Range & {$[5 ; 27]$} & {$[3 ; 40]$} & {$[3 ; 40]$} \\
\hline
\end{tabular}

a The partial Mayo Score consists of the Mayo score minus the sigmoidoscopy subscore; the partial score ranges from 0 to 9 , with higher scores indicating more active disease. ${ }^{b}$ Data show 1 patient with a partial Mayo Score of 11 at baseline. This appears to be have been an enrolment error (see also Discussion section).

as detected by a mobile real-time monitoring device used during the titration period from day 1 to 12 (Fig. 1).

\section{Efficacy Analysis}

The primary efficacy variable was based on clinical remission, defined as a partial Mayo Score of 0 or 1 and a modified Baron Score of 0 or 1 with subscore of 0 for rectal bleeding. All patients who completed at least 28 days of treatment (i.e., visit 6) were included in the efficacy analysis. Patients who discontinued after at least 28 days of treatment due to lack of efficacy were considered nonresponders. A total of $14 \mathrm{KRP} 203$-treated patients and 8 placebo-treated patients qualified for the primary efficacy analysis. At week 8, clinical remission occurred in 2 of 14 patients (14\%) treated with $1.2 \mathrm{mg}$ KRP203 as compared with 0 of $8(0 \%)$ who received placebo. With a $17.5 \%$ probability in favour of $\mathrm{H} 1$ and $83.7 \%$ probability of rejecting $\mathrm{H} 0$, the primary end point of the study was not reached. The criteria for reaching the primary end point required a probability of at least $50 \%$ in favour of $\mathrm{H} 1(20$ percentage points more responders in the active group than in the placebo group) and $95 \%$ probability against $\mathrm{HO}$ (difference between active and placebo $>0$ ). The results may suggest that KRP203 treatment is superior to placebo, but clearly inferior to the minimal clinically relevant threshold of $20 \%$ (H1) (Fig. 3), and without statistical significance. Partial Mayo Scores at baseline, week 8, and day 5 are shown in Figure 4.

Mucosal healing was classified using the modified Baron Score at baseline and day 56, with an optional additional assessment at week 4 . Due to the relatively high rate of early discontinuation, the results obtained for the mod- 
Table 2. AEs overall and frequently affected SOCs

\begin{tabular}{llll}
\hline & $\begin{array}{l}\text { KRP203 1.2 mg } \\
n=17, n(\%)\end{array}$ & $\begin{array}{l}\text { Placebo } \\
n=10, n(\%)\end{array}$ & $\begin{array}{l}\text { Total } \\
n=27, n(\%)\end{array}$ \\
\hline Patients with AEs & $15(88.2)$ & $9(90.0) 24$ & $24(88.9)$ \\
\hline SOC & & & \\
Gastrointestinal disorders & $7(41.2)$ & $4(40.0)$ & $11(40.7)$ \\
Nervous system disorders & $5(29.4)$ & $2(20.0)$ & $7(25.9)$ \\
General disorders and administration site conditions & $5(29.4)$ & $1(10.0)$ & $6(22.2)$ \\
Musculoskeletal and connective tissue disorders & $4(23.5)$ & $2(20.0)$ & $6(22.2)$ \\
Infections and infestations & $4(23.5)$ & $1(10.0)$ & $5(18.5)$ \\
Investigations & $0(0.0)$ & $4(40.0)$ & $4(14.8)$ \\
Skin and subcutaneous tissue disorders & $3(17.6)$ & $1(10.0)$ & $4(14.8)$ \\
Eye disorders & $2(11.8)$ & $1(10.0)$ & $3(11.1)$ \\
Blood and lymphatic system disorders & $2(11.8)$ & $0(0.0)$ & $2(7.4)$ \\
Respiratory, thoracic, and mediastinal disorders & $2(11.8)$ & $0(0.0)$ & $2(7.4)$ \\
Vascular disorders & $1(5.9)$ & $1(10.0)$ & $2(7.4)$ \\
Endocrine disorders & $0(0.0)$ & $1(10.0)$ & $1(3.7)$ \\
Hepatobiliary disorders & $1(5.9)$ & $0(0.0)$ & $1(3.7)$ \\
Metabolic and nutrition disorders & $0(0.0)$ & $1(10.0)$ & $1(3.7)$ \\
Psychiatric disorders & $0(0.0)$ & $1(10.0)$ & $1(3.7)$ \\
Surgical and medical procedures & $1(5.9)$ & $0(0.0)$ & $1(3.7)$ \\
\hline
\end{tabular}

AEs, by SOC, are presented in descending order of frequency in both groups together (= "Total"). AEs, adverse event; SOC, system organ class.

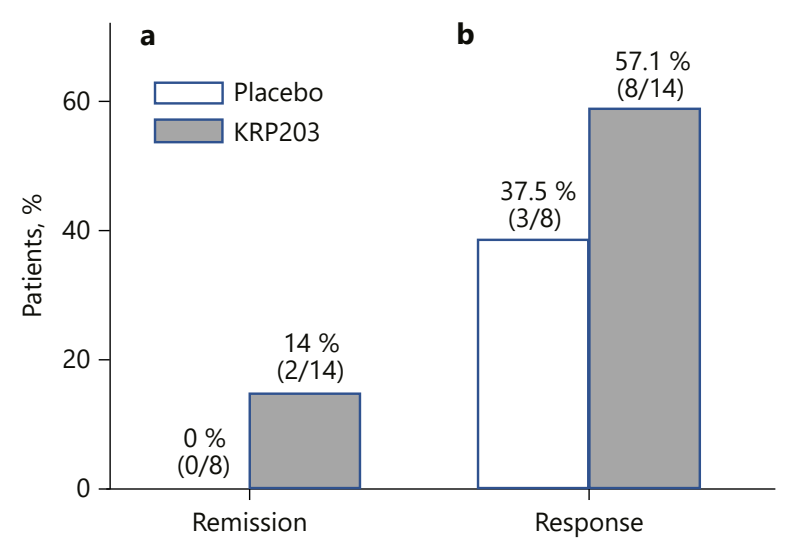

Fig. 3. Efficacy outcomes at week 8 of KRP203 as induction therapy. Panel a shows the percentage of patients in clinical remission (defined as a partial Mayo Score of 0 or 1 together with a modified Baron Score of 0 or 1 with no macroscopic bleeding, i.e., score 0 in the "rectal bleeding" dimension of the partial Mayo Score) at week 8 (the primary outcome). Panel $\mathbf{b}$ shows the percentage of patients showing a partial response, defined as a reduction of partial Mayo Score of at least 3 points from baseline to week 8 . ified Baron Score after 56 days were derived from only 10 patients (out of 17 at baseline) in the KRP203 group and 5 (out of 10 at baseline) in the placebo group. Therefore, any conclusions drawn from the endoscopic assessments are of limited value.

The mean reduction in corticosteroid dose was another key secondary end point. The frequency of steroid dose reduction during the study was found to be low, with 3 patients on KRP203 (17.6\%) and 1 patient on placebo (10\%) reducing their steroid dose (data not shown).

In the KRP203 cohort, a time-dependent decrease in ALC was observed, as expected for an S1P1 receptor modulator, reaching a nadir $\sim 2$ weeks after treatment initiation (Fig. 5). In 1 patient, ALC dropped below the safety threshold of $0.2 \times 10^{9} / \mathrm{L}$, as a result of which this individual's study participation was discontinued. Leucocyte subsets were also analysed during the study to investigate the impact of KRP203 on different cell populations; the subsets included $\mathrm{T}$ cells, natural killer cells and monocytes, B cells, plasma cells, and dendritic cells. The percentage of $\mathrm{CD} 3+$ ( $\mathrm{T}$ cells) in the total leucocyte population was observed to be diminished at day 28 compared to baseline. CD4+ T cells were more reduced than CD8+ $\mathrm{T}$ cells. In addition, a decrease in $\mathrm{B}$ cells occurred during KRP203 treatment (baseline vs. day 28). 


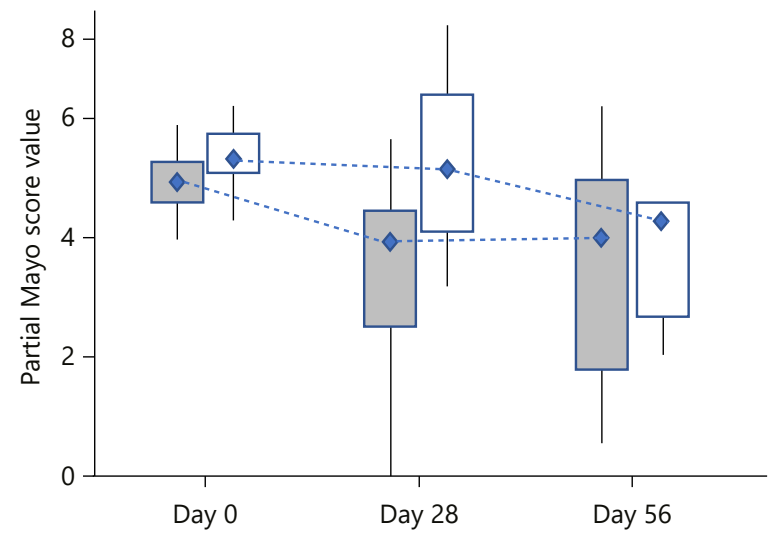

Fig. 4. Partial Mayo Scores at baseline, week 8, and day 56.

\section{Discussion}

The present phase II trial was a placebo-controlled, parallel-group, multicentre study evaluating the $\mathrm{PK}, \mathrm{PD}$, and efficacy of KRP203 in patients with moderately active UC and inadequate response to 5-ASA treatment. Therapy with KRP203 at a once-daily oral dose of $1.2 \mathrm{mg}$ resulted in higher rates of clinical remission at week 8 than were observed in patients treated with placebo (14 vs. $0 \%$ ). Although, at least partly due to the high rate of early termination, these results are not significant, they appear remarkably similar to those of the recently published $\mathrm{TOUCH}-$ STONE trial investigating the efficacy of ozanimod, another S1P1 agonist, in a comparable patient population. Regrettably, however, substantially different parameters were chosen in the TOUCHSTONE trial to define clinical remission (total Mayo Score $\leq 2$, with no individual subscore $>1$ ). Due to this discrepancy, and to the use of a different drug dosage (ozanimod was given at a dose of $1 \mathrm{mg}$ / day, whereas the current study took a dose of $1.2 \mathrm{mg} /$ day KRP203), a direct comparison of the efficacy results of both studies is not possible. Compared with the placebo cohort, ozanimod at a once-daily oral dose of $1 \mathrm{mg}$ induced clinical remission at week 8 in $16 \%$ versus $6 \%$ of patients and clinical response at week 8 in $57 \%$ versus 37\% [5].

Importantly, the sufficiency of PD effects of KRP203 at the final daily dose of $1.2 \mathrm{mg}$ is further supported by a reduction of the S1P1-typical PD-marker ALC to a level of $37 \%$ at 8 weeks compared to baseline (in the ozanimod study, ALC was reduced to $49 \%$ at the same time point) [5]. This would suggest that KRP203 may exhibit a PD potency comparable to that of ozanimod.

S1P Receptor Agonist KRP203 in

Ulcerative Colitis
KRP203 was generally safe and well tolerated, and AEs were similar in the active study drug and placebo cohorts. No severe or dose-limiting toxicities were observed. Importantly, no cardiac AEs were reported in the KRP203 group, demonstrating that the utilised dose titration regimen was able to mitigate bradycardia.

Nevertheless, the study failed its primary objective of a remission rate at least $20 \%$ higher for KRP203 than placebo at week 8 . At the first IA of 22 evaluable patients, the predefined futility criteria were met, as a result of which the trial was terminated prematurely. In the final analysis, while 2 patients achieved remission in the KRP203 cohort versus none in the placebo group, the efficacy was not considered sufficient to continue the study. Bearing in mind the 2 patients in remission, a beneficial effect of KRP203 cannot be completely ruled out. Subgroup analyses of patients with or without concomitant treatment with corticosteroids or previous treatment with antiTNF- $\alpha$ therapy did not reveal any major differences.

The efficacy biomarkers CRP, erythrocyte sedimentation rate, and faecal calprotectin failed to yield any conclusive results, not least due to the small patient population and the wide intrapatient variability of these parameters, especially faecal calprotectin: Whereas serum CRP $>5 \mathrm{mg} / \mathrm{L}$ or documented endoscopic evidence of mucosal ulcerations was an inclusion criterion for the study, there was no similar specification for concentrations of faecal calprotectin.

The trial had some limitations. First, the primary end point of clinical remission was not met. It was not possible to obtain more conclusive data (as originally planned in a total study population of 60 patients), since the interim results (including only 27 patients) fulfilled the futility criteria defined for premature termination of the study. Second, the time point (week 8) chosen for efficacy evaluation may not be long enough for drugs targeting lymphocyte trafficking, a possibility supported by the enhanced benefits seen with anti-trafficking agents such as Vedolizumab [16]. Furthermore, at this early time point, conclusions regarding efficacy may potentially be confounded by the fact that only 3 patients on KRP203 (vs. 1 on placebo) were able to taper corticosteroids, which was permitted as per study protocol after 2 weeks of study medication. In line with this, the TOUCHSTONE trial of Sandborn et al. [5] revealed an increased proportion of ozanimod-treated patients in remission at week $32 \mathrm{com}$ pared to week 8 , leading the authors to suggest that extended treatment may be associated with enhanced efficacy. Third, according to data shown in the study report, 1 of the patients had a partial Mayo Score at baseline of

Inflamm Intest Dis 2020;5:180-190 187 
Fig. 5. Individual (a) and mean change (b) in absolute lymphocyte count \pm SEM in subjects receiving $1.2 \mathrm{mg} /$ day KRP203 for 28 days after dose escalation of KRP203 $(0.3 \mathrm{mg} /$ day on days $1-4 ; 0.6 \mathrm{mg} /$ day on days 5-8; and $0.9 \mathrm{mg} /$ day on days $9-12$ ) versus placebo. Baseline for all trough samples is defined as the day $0,-24$-h ALC values. ALC, absolute lymphocyte count; SEM, standard error of the mean.
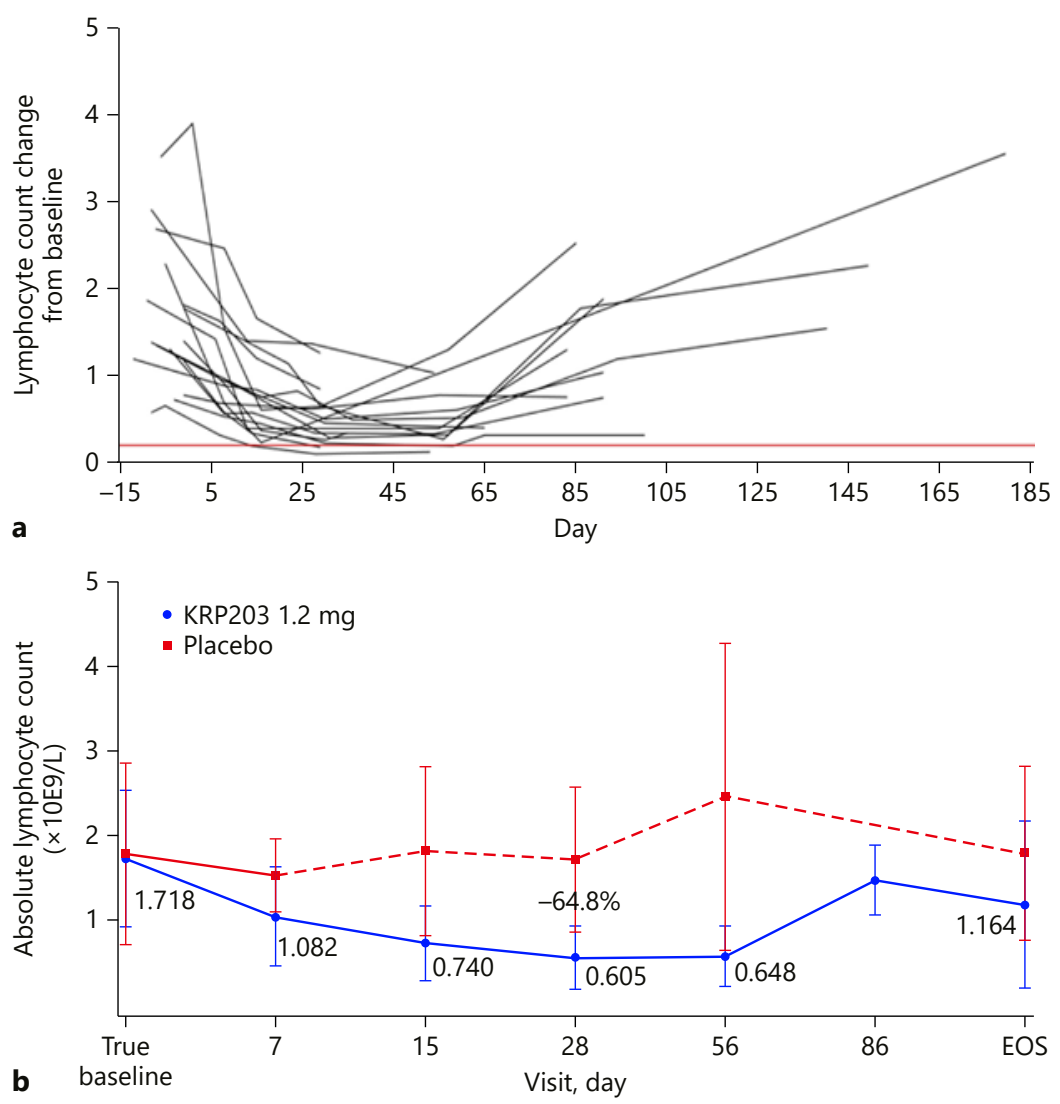

11, whereas the maximum score allowed according to the inclusion criteria was 9. This should have led to the patient's exclusion from the study. Unfortunately, we were unable to rectify this issue. Fourth, while a limit of $40 \mathrm{mg} /$ day was applied and patients were stratified according to steroid intake (yes/no) at baseline, the inclusion/exclusion criteria of the present study failed to define a specific period during which the oral steroid dose was required to have been stable prior to baseline. Furthermore, no data are available regarding prior UC therapies of the study subjects. Finally, as noted above, given the relatively brief duration of observation and the small number of patients evaluated, a meaningful safety evaluation of KRP203 was not possible. While we acknowledge the evident shortcomings of the study, we felt it important to make its findings available to a wider readership - especially in the light of the fact that results of the phase 1 studies of KRP203 have not been published.

Although a marked reduction in peripheral blood lymphocyte numbers was noted in all KRP203-treated pa- tients, this was not found to correlate with major clinical improvement. Further exploratory analyses are in progress to improve mechanistic understanding of the mode of action of KRP203 in UC. These will be summarised in a separate report.

In conclusion, in this preliminary trial, KRP203 at a dose of $1.2 \mathrm{mg}$ was safe and well tolerated by patients with moderately active UC. There were no instances of bradycardia or cardiac AEs. However, the trial was not large enough or of sufficiently long duration to enable a meaningful assessment of the safety of the drug. KRP203 was associated with a slightly higher rate of clinical remission than placebo treatment. Even though the study was terminated prematurely, the results may suggest that KRP203 treatment is superior to placebo. While in principle, therefore, the potential effectiveness of the mode of action of 2 different S1P1-agonists has now been shown in similar patient populations, due to the delayed action of the drug type, together with the stringently chosen efficacy and futility criteria in the KRP203 study, which played a 
role in its early discontinuation, no significant results could be obtained which would have allowed a direct comparison of the 2 substances. Larger randomised controlled trials designed to include a maintenance and/or extension phase may be considered for conclusive assessment of the efficacy and tolerability of KRP203 in patients with UC.

\section{Acknowledgements}

The authors extend their grateful thanks to Janet Collins (Interdisciplinary Crohn-Colitis Centre Rhein-Main, Frankfurt, Germany) for editing and proofreading the manuscript, and to all the participating study centres and investigators.

\section{Statement of Ethics}

The protocol (CKRP203A2201) was approved by the Independent Ethics Committee for human research of the Ärztekammer Nordrhein (North Rhine Westphalia) and the local institutional review board responsible for each trial centre. The trial was conducted in accordance with the World Medical Association Declaration of Helsinki and the ethical principles of Good Clinical Practice according to the International Conference on Harmonization (ICH) Harmonized Tripartite Guideline. The trial was registered at Clinicaltrials.gov (identifier NCT01375179) prior to patient enrolment, with the EudraCT number 2010-019970-33. All patients were personally informed about all aspects of the trial including possible risks of treatment and data protection measures, and all provided written informed consent prior to screening.

\section{Conflict of Interest Statement}

H.H.R. has received expenses from Novartis for attendance of the investigator meeting for this study and has no other conflicts of interest. W.K. has received consultancy fees from Falk, Ferring, Genetic Analysis, Istitut Allergosan, Nikkiso, and Otsuka, and lecturing fees from Abbvie, Ardeypharm, and Tillotts. J.S. has received consultancy fees from Abbvie, Fresenius-Kabi, Immundiagnostik, MSD, Pharmacosmos, Takeda, GI Dynamics, and Vifor; lecturing fees from Abbvie, Falk Foundation, Ferring, Immundiagnostik, MSD, Pharmacosmos, Takeda, Thermofischer, GI Dynamics, and Vifor; and payment for manuscript preparation from Abbvie, Falk Foundation, GI Dynamics, and MSD. P.L.L. has no conflicts of interest to declare. P.J. and P.G. are employees of Novartis. L.C. is an employee and stockholder of Novartis. P.M. is an employee and stockholder of Novartis Institutes of Biomedical Research. F.M. is an employee and stockholder of Novartis Bioventures Ltd. G.R. has consulted to Abbot, Abbvie, Augurix, Boehringer, Calypso, FALK, Ferring, Fisher, Genentech, Essex/MSD, Novartis, Pfizer, Phadia, Roche, UCB, Takeda, Tillots, Vifor, Vital Solutions, and Zeller; received speaker's honoraria from Astra Zeneca, Abbott, Abbvie, FALK, MSD, Phadia, Tillots, UCB, and Vifor; and received educational and research grants from Abbot, Abbvie, Ardeypharm, Augurix, Calypso, Essex/MSD, FALK, Flamentera, Novartis, Roche, Takeda, Tillots, UCB, and Zeller. G.A. has received expenses from Novartis for meetings in connection with this study; consultancy fees from Janssen, Takeda, and Abbvie; and lecturing fees from Abbvie and Pfizer.

\section{Funding Sources}

This study was funded by Novartis. Employees of the sponsor were involved in writing the statistical analysis plan, monitoring data collection, analysing the study results, and preparing the manuscript (see Author Contributions, below). The authors declare that this manuscript was prepared entirely independently of the interests of the sponsor.

\section{Author Contributions}

H.H.R. and J.S. contributed to the study protocol design, study implementation, and data interpretation, and drafted and finalised the manuscript. W.K. was principal investigator of the study and contributed to the study protocol design, study implementation, data interpretation, and manuscript writing. P.G. contributed to the study protocol design, study implementation, data interpretation, and manuscript writing. G.A., G.R., and P.L.L. as clinical trial leaders monitored data collection, interpreted study findings, and assisted with manuscript writing. F.M., P.M., P.J., L.C., and P.G. wrote the statistical analysis plan, monitored data collection, and analysed the study results. F.M. and P.G. assisted in editing the manuscript draft. All authors made a collective decision to submit the manuscript for publication, and all read and approved the final version of the manuscript prior to submission. All authors vouch for the veracity of the data and analyses reported and for the adherence of the trial to the protocol. Confidentiality agreements were in place between the sponsor and all authors.

\section{Conference Presentation}

Preliminary data from this trial were first presented in 2016 at the ECCO Congress in Amsterdam and the DDW in San Diego.

References

1 Mandala S, Hajdu R, Bergstrom J, Quackenbush E, Xie J, Milligan J, et al. Alteration of lymphocyte trafficking by sphingosine1-phosphate receptor agonists. Science. 2002 Apr 12;296(5566):346-9.

2 Marsolais D, Rosen H. Chemical modulators of sphingosine-1-phosphate receptors as barrier-oriented therapeutic molecules. Nat Rev Drug Discov. 2009 Apr;8(4):297307.

3 Schwab SR, Cyster JG. Finding a way out: lymphocyte egress from lymphoid organs. Nat Immunol. 2007 Dec;8(12):1295-301. 
4 Khatri BO. Fingolimod in the treatment of relapsing-remitting multiple sclerosis: longterm experience and an update on the clinical evidence. Ther Adv Neurol Disord. 2016 Mar; 9(2):130-47.

5 Sandborn WJ, Feagan BG, Wolf DC, D'Haens G, Vermeire S, Hanauer SB, et al. Ozanimod induction and maintenance treatment for ulcerative colitis. N Engl J Med. 2016 May 5; 374(18):1754-62.

6 Fujishiro J, Kudou S, Iwai S, Takahashi M, Hakamata Y, Kinoshita M, et al. Use of sphingosine-1-phosphate 1 receptor agonist, KRP203 , in combination with a subtherapeutic dose of cyclosporine a for rat renal transplantation. Transplantation. 2006 Sep 27;82(6):804-12.

7 Shimizu H, Takahashi M, Kaneko T, Murakami T, Hakamata Y, Kudou S, et al. KRP-203, a novel synthetic immunosuppressant, prolongs graft survival and attenuates chronic rejection in rat skin and heart allografts. Circulation. 2005 Jan 18;111(2):222-9.
8 Khattar M, Deng R, Kahan BD, Schroder PM, Phan T, Rutzky LP, et al. Novel sphingosine1-phosphate receptor modulator KRP203 combined with locally delivered regulatory $\mathrm{T}$ cells induces permanent acceptance of pancreatic islet allografts. Transplantation. 2013 Apr 15;95(7):919-27.

9 Song J, Matsuda C, Kai Y, Nishida T, Nakajima K, Mizushima T, et al. A novel sphingosine 1-phosphate receptor agonist, 2-amino2-propanediol hydrochloride (KRP-203), regulates chronic colitis in interleukin-10 gene-deficient mice. J Pharmacol Exp Ther. 2008 Jan;324(1):276-83.

10 Huwiler A, Pfeilschifter J. New players on the center stage: sphingosine 1-phosphate and its receptors as drug targets. Biochem Pharmacol. 2008 May 15;75(10):1893-900.

11 Baron, JH, Connell AM, Lennard-Jones JE. Variation between observers in describing mucosal appearances in proctocolitis. BMJ. 1964;1(5375):89-92.

12 Fisch R, Jones I, Jones J, Kerman J, Rosenkranz GK, Schmidli H. Bayesian design of proof-of-concept trials. Ther Innov Regul Sci. 2015 Jan;49(1):155-62.
13 Feagan BG, Greenberg GR, Wild G, Fedorak RN, Paré P, McDonald JW, et al. Treatment of ulcerative colitis with a humanized antibody to the alpha4beta7 integrin. $\mathrm{N}$ Engl Med. 2005 Jun 16;352(24):2499-507.

14 Rutgeerts P, Sandborn WJ, Feagan BG, Reinisch W, Olson A, Johanns J, et al. Infliximab for induction and maintenance therapy for ulcerative colitis. N Engl J Med. 2005 Dec 8; 353(23):2462-76

15 Van Assche G, Sandborn WJ, Feagan BG, Salzberg BA, Silvers D, Monroe PS, et al. Daclizumab, a humanised monoclonal antibody to the interleukin 2 receptor (CD25), for the treatment of moderately to severely active ulcerative colitis: a randomised, double blind, placebo controlled, dose ranging trial. Gut. 2006 Nov;55(11):1568-74.

16 Feagan BG, Rutgeerts P, Sands BE, Hanauer S, Colombel JF, Sandborn WJ, et al. Vedolizumab as induction and maintenance therapy for ulcerative colitis. N Engl J Med. 2013 Aug 22;369(8):699-710 